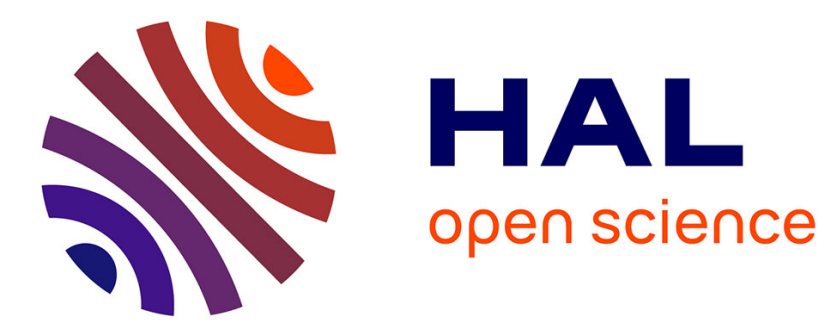

\title{
Boosting the Efficiency of Byzantine-tolerant Reliable Communication
}

\author{
Silvia Bonomi, Giovanni Farina, Sébastien Tixeuil
}

\section{To cite this version:}

Silvia Bonomi, Giovanni Farina, Sébastien Tixeuil. Boosting the Efficiency of Byzantine-tolerant Reliable Communication. [Technical Report] Sorbonne Université, CNRS, LIP6, F-75005 Paris, France; Sapienza Università di Roma, Rome, Italy. 2020. hal-02960087

\section{HAL Id: hal-02960087 https://hal.science/hal-02960087}

Submitted on 8 Oct 2020

HAL is a multi-disciplinary open access archive for the deposit and dissemination of scientific research documents, whether they are published or not. The documents may come from teaching and research institutions in France or abroad, or from public or private research centers.
L'archive ouverte pluridisciplinaire HAL, est destinée au dépôt et à la diffusion de documents scientifiques de niveau recherche, publiés ou non, émanant des établissements d'enseignement et de recherche français ou étrangers, des laboratoires publics ou privés. 


\title{
Boosting the Efficiency of Byzantine-tolerant Reliable Communication *
}

\author{
Silvia Bonomi ${ }^{1}$, Giovanni Farina ${ }^{2,1}$, and Sébastien Tixeuil ${ }^{2}$ \\ 1 Sapienza Università di Roma, Rome, Italy \\ bonomi@diag. uniroma1.it \\ 2 Sorbonne Université, CNRS, LIP6, F-75005 Paris, France \\ giovanni.farina@lip6.fr, sebastien.tixeuil@lip6.fr \\ Technical Report
}

\begin{abstract}
Reliable communication is a fundamental primitive in distributed systems prone to Byzantine (i.e. arbitrary, and possibly malicious) failures to guarantee integrity, delivery and authorship of messages exchanged between processes. Its practical adoption strongly depends on the system assumptions. One of the most general (and hence versatile) such hypothesis assumes a set of processes interconnected through an unknown communication network of reliable and authenticated links, and an upper bound on the number of Byzantine faulty processes that may be present in the system, known to all participants.

To this date, implementing a reliable communication service in such an environment may be expensive, both in terms of message complexity and computational complexity, unless the topology of the network is known. The target of this work is to combine the Byzantine fault-tolerant topology reconstruction with a reliable communication primitive, aiming to boost the efficiency of the reliable communication service component after an initial (expensive) phase where the topology is partially reconstructed. We characterize the sets of assumptions that make our objective achievable, and we propose a solution that, after an initialization phase, guarantees reliable communication with optimal message complexity and optimal delivery complexity.
\end{abstract}

Keywords: Reliable communication · Byzantine fault tolerance · Topology reconstruction

\section{Introduction}

Reliable communication primitives are fundamental building blocks for a distributed system, guaranteeing the eventual delivery of all messages sent by cor-

\footnotetext{
* This work was performed within Project ESTATE (Ref. ANR-16-CE25-0009-03), supported by French state funds managed by the ANR (Agence Nationale de la Recherche) and it has been partially supported by the INOCS Sapienza Ateneo 2017 Project (protocol number RM11715C816CE4CB). Giovanni Farina wishes to thank Université Franco-Italienne/Universitá Italo-Francese (UFI/UIF) for supporting his mobility through the Vinci grant 2018.
} 
rect processes to their intended receivers. Their employment is particularly relevant when a fraction of processes may suffer arbitrary failures i.e., they are Byzantine and may deviate from the protocol by dropping messages, altering their content, or generating spurious messages.

The availability of a reliable communication primitive strongly depends on the system behavior and on its capability to match the set of assumptions required to ensure the correctness of the reliable communication specification. In particular, it has been shown that such a primitive can be efficiently implemented when every process can directly exchange messages with every other [4, also in presence of a bounded and known number of Byzantine processes. However, full connectivity is a strong assumption in large networks and it results impractical whenever scalability is envisioned.

When considering multi-hop networks i.e., systems where every process can communicate directly only with a subset of the others, several results exist to build a Byzantine-tolerant reliable communication primitive. In this paper, we are interested in the solutions designed for multi-hop networks where the topology is not known to the participants. In this context, 7] defined a solution working under the assumption that processes are sufficiently connected. However, providing a reliable communication service in such a general environment may mandate a huge amount of messages and may require very high computational power. Those complexity issues can somewhat be reduced to a tractable problem when either the entire network topology is known to all the processes [7] or it satisfies specific topological requirements [17]. Thus, a naive approach to build an efficient reliable communication primitive is to act in two steps: (i) run a topology reconstruction algorithm to infer the network graph and (ii) use an efficient reliable communication protocol for known network on the reconstruction just inferred. Unfortunately, Byzantine fault-tolerant topology reconstruction has been proved difficult [16, and the final topology inferred does not perfectly match the real one. Besides, correct peers may end the topology reconstruction algorithm by obtaining different network graphs.

Given a network topology $G$ unknown to processes, our goal in this paper is to detail how properly combine the two steps of the described naive approach and to study the set of conditions that $G$ must satisfy to correctly support it. The rationale of this work is that the high topology reconstruction overhead only needs to be paid once, afterwards, reliable communication can be achieved efficiently (otherwise, it would have remained always extremely expensive). The main difficulty is to ensure that discrepancies in the topology reconstructions do not hinder the proper functioning of the reliable communication system. Our work builds upon two reliable communication protocols (DolevR and DolevU [7]), and a topology reconstruction one (Explorer [16]). In more detail, we characterize the sets of assumptions that make our objective achievable, and we propose a solution that, after an initialization phase, guarantees reliable communication with optimal message complexity and delivery complexity. 


\section{Related Works}

Several solutions have been proposed in the literature to build Byzantine-tolerant reliable communication primitives. A seminal contribution was provided by Dolev [7, assuming (i) processes interconnected through a possibly multi-hop communication network (ii) and an upper bound $f$ on Byzantine faulty processes present in the system (globally bounded failure model). Dolev proved that a $(2 f+1)$-connected network is required to build a reliable communication primitive in presence of $f$ Byzantine participants i.e., the node connectivity of the communication network must be greater than twice the maximum estimated number of faulty processes. Dolev proposed two protocols working with different assumptions on the knowledge of the network topology by participating processes. More precisely, the lack of topology knowledge impacts both the message complexity (i.e., the number of messages exchanged in the system during a reliable communication instance) and the delivery complexity (i.e., the computational complexity of the procedure used to validate a message) of the protocol. The Dolev's protocol for unknown networks was recently revised to reduce its message complexity [3]. To the best of our knowledge, no other contribution addressed the reliable communication problem in the globally bounded failure model without considering stronger assumptions. When moving to the locally bounded failure model (where at most $f$ Byzantine failures are present in the neighborhood of every process) other approaches have been defined [18. The Certified Propagation Algorithm (CPA) was proposed as a reliable communication protocol by Pelc and Peleg, and it has been proven optimal, for the number of faulty processes that can be simultaneously tolerated, in the locally bounded failure model [17. Let us note that, either assuming a globally or locally bounded failure model, a dense communication network is required to enable reliable communication in a distributed system. For this reason, weaker primitives have been defined, allowing a (small) part of correct processes to either deliver spurious messages (i.e. messages not generated by their claimed author) or to never deliver a valid message [13:12]14]. These weaker versions enable almost reliable communication also on sparse communication networks.

All the aforementioned solutions do not necessarily rely on digital signatures or other cryptographic primitives. Indeed, the goal of Byzantine-tolerant algorithms is to withstand (computationally) unbounded adversaries that are potentially able to solve (computationally hard) problems on which cryptographic primitives are based upon. Nevertheless, links are assumed to be authenticated and reliable, so if $u$ and $v$ are linked, every message $v$ received from $u$ has been previously sent by $u$. Notice that cryptographic primitives are not necessary to implement authenticated links [20].

The Byzantine fault-tolerant topology reconstruction problem has been analyzed by Nesterenko and Tixeuil [16] assuming the globally bounded failure model. Then, temporary arbitrary faults have been considered by Dolev et al., defining a self-stabilizing Byzantine-tolerant solution [8]. 


\section{Preliminaries}

\subsection{System Model}

We consider an asynchronous distributed system [4] composed by a set $P$ of $n$ processes, each associated with a unique identifier i.e., $P=\left\{p_{1}, p_{2}, \ldots, p_{n}\right\}$.

Failure Model. We consider the globally bounded Byzantine failure model, namely we assume that inside the system there might be at most $f$ Byzantine faulty processes. All other peers are assumed correct. Let us note that the identity of Byzantine processes is not known to correct ones.

Messages and Communication. Processes communicate by exchanging messages on top of a communication network made of reliable and authenticated links [4. It means that messages cannot be lost or altered during their transmission and that the identity of their sender cannot be forged. Such communication network is abstracted by an undirected graph $G=(P, E)$ where the set of nodes corresponds the set of processes participating in the system and the set of edges $E$ contains an element $e_{i, j}$ for each existing link connecting two processes $p_{i}$ and $p_{j}$. We assume the node connectivity $k$ of $G$ greater than twice the number of the potentially faulty processes i.e., $k>2 f 3$

On top of the communication network, two alternative primitives are available: unicast $(U L)$ and local broadcast $(L B L)$ links [1211]: the former interconnect single pairs of processes $p_{i}, p_{j}$; the latter attach a process $p_{i}$ to many others, such that if a message is sent by $p_{i}$ then it is received by all of its neighbors, thus preventing a faulty process to equivocate (i.e., to transmit conflicting messages to different neighbors).

We assume that processes are unaware about the topology of the communication network, namely the graph $G$ : they either know the identifier of the peers they have a link with (known neighborhood i.e., $K N$ assumption) or they have no knowledge about (unknown neighborhood i.e., UN assumption).

We refer with source to the advertised author of a message, and with sender to the process that is sending a message through a link.

\subsection{Problem Specification: Reliable Communication}

We investigate the reliable communication problem between a source process $p_{s}$ and a target process $p_{t}$. Informally, when addressing this problem, the goal is to define a distributed protocol able to deliver only the messages generated by a correct source to every correct process in the system.

Let us note that, in the literature, the term message is commonly used instead of content when formalizing a problem specification based on message exchanges. However, several messages carrying the same payload can be diffused in a system to solve the reliable communication problem. Therefore, for ease of presentation, we will refer with content to the payload diffused by a process and with message

\footnotetext{
${ }^{3}$ It is not possible otherwise to achieve reliable communication in the system model we are considering [7].
} 
to union of a content and the protocol specific overhead.

More formally, we will say that a protocol solves the reliable communication problem if, for every pair of processes $p_{s}$ and $p_{t}$ in the system, both the following conditions are satisfied:

- (Safety) if $p_{t}$ is correct and it delivers a content $m$ from $p_{s}$, then $p_{s}$ previously sent $m$;

- (Liveness) if $p_{s}$ and $p_{t}$ are both correct and $p_{s}$ sends a content $m$ to $p_{t}$, then $p_{t}$ eventually delivers $m$ from $p_{s}$.

We refer with spurious content to one not sent by its claimed source (i.e. a content initially diffused by some Byzantine process).

\subsection{Evaluation Metrics}

We will evaluate the solutions to the reliable communication problem in terms of (i) message complexity i.e., the number of messages that the protocol generates to solve the problem and (ii) delivery complexity i.e., the computational complexity of the procedure that allows a target process $p_{t}$ to decide if a content can be delivered or not.

\subsection{The Topology Reconstruction Problem}

Given an unknown network $G$ of correct and Byzantine faulty processes, the aim of a distributed protocol addressing the topology reconstruction problem is to enable all correct processes to reconstruct a subset of the topology of the communication network $G$. Such a reconstruction $G_{i}$ is expected to be as complete as possible. The nodes of the communication network $G$ can be partitioned in correct and faulty, and its edges in correct, one-faulty and two-faulty, respectively interconnecting two correct processes, a correct process and a faulty one, and two Byzantine processes. Likewise, the nodes and edges of a topology reconstruction $G_{i}$ can be either real or spurious, respectively mapping or not nodes and edges in $G$.

\subsection{Basic Definitions}

For the sake of presentation, this section recalls some definitions and results coming from graph theory [5] that will be employed in this work.

Let us consider an undirected graph $G=(V, E)$. A path $\mathcal{P}$ is a sequence of nodes with no repetition i.e., $\mathcal{P}=\left[v_{1}, v_{2}, \ldots, v_{m}\right]$ (with $v_{i} \in V$ ) such that for each pair of adjacent elements $v_{i}, v_{i+1}$ there exists an edge $e_{i, i+1} \in E$. The first and last elements of a path are referred with endpoints.

A pair of nodes $v_{i}, v_{j} \in V$ is connected if there exists at least one path $\mathcal{P}_{i, j}$ between them in $G$, it is disconnected otherwise. Given two nodes $v_{i}$ and $v_{j}$, many paths between them may exist. Given a set of paths $\mathcal{P}_{i, j}^{1}, \mathcal{P}_{i, j}^{2}, \ldots, \mathcal{P}_{i, j}^{x}$ between two nodes $v_{i}$ and $v_{j}$ they are said node disjoint if they share no vertex except for their endpoints. 
We refer with $\Pi_{i, j}$ to a disjoint paths solution between nodes $v_{i}$ and $v_{j}$, i.e. a set of node disjoint paths having $v_{i}$ and $v_{j}$ as endpoints. The local node connectivity $\kappa_{i, j}$ between two nodes $v_{i}, v_{j}$ is the minimum number of nodes that has to be removed from $G$ to disconnect $v_{i}$ from $v_{j}$. The node connectivity of a graph is the minimum value $k$ for the local node connectivity $\kappa_{i, j}$ (i.e., $\left.k=\min \left(\kappa_{i, j}\right), \forall v_{i}, v_{j} \in V\right)$. A graph having node connectivity greater or equal than $k$ is said $k$-connected graph. The local node connectivity between two nodes is equal to the maximum number of disjoint paths that exist between them (Menger theorem [15]). It is possible to compute a disjoint paths solution $\Pi_{i, j}$ between two nodes $v_{i}, v_{j}$ of maximum size (namely $\kappa_{i, j}$ ) with a deterministic algorithm with computational complexity polynomial in the size of the graph 966. In the following, we will consider every disjoint paths solution $\Pi_{i, j}$ always of maximum size $\kappa_{i, j}$.

A generalized wheel [19, denoted by $W(a, b)$, is an undirected graph of order $a+b$ obtained from the disjoint union of a complete graph $K_{a}$ and a cycle $C_{b, b \geq 3}$ by adding edges joining each vertex of $K_{a}$ to all nodes of $C_{b}$.

\section{Dolev Protocols}

Dolev [7] identified the necessary and sufficient conditions to solve reliable communication in the system model we consider.

Remark 1. The reliable communication problem can be solved if and only if the node connectivity of the communication graph is greater than $2 f$ i.e., $k>2 f$.

Dolev provided two protocols that work under different assumptions on the (partial) knowledge that processes have about the network topology.

\subsection{Dolev's Routed Protocol (DolevR)}

DolevR is a protocol solving reliable communication in routed-networks [7, i.e. systems where all messages are relayed over (and only) fixed and known paths. Specifically, processes employing DolevR route contents between each pair of process $p_{i}, p_{j}$ over $2 f+1$ disjoint paths $\Pi_{i, j}$. The reliable and authenticated links restrict the capabilities of faulty processes, allowing them to diffuse spurious contents through at most $f$ paths of any $\Pi_{i, j}$. The assumption of a $(2 f+1)$ connected network guarantees that at least $f+1$ paths of any $\Pi_{i, j}$ are fault-free (i.e. they do not pass through any faulty processes). A process $p_{j}$ employing DolevR delivers a content $m$ from a process $p_{i}$ if it is received through at least $f+1$ routes of $\Pi_{i, j}$.

Protocol Complexity. The message complexity of DolevR is linear in the size of the network, whereas its delivery complexity is linear in the number of maximum assumed faults, as detailed in the following Lemmas.

Lemma 1. DolevR solves the reliable communication problem with $O(n)$ message complexity. 
Proof. A source process $p_{s}$ in DolevR routes every content over $2 f+1$ disjoint paths $\Pi_{s, t}$ to the target process $p_{t}$ : $p_{s}$ sends $2 f+1$ messages whereas all other processes in $\Pi_{s, t}$ but $p_{s}$ sends one message. It follows that the number of messages exchanged is $O(n)$.

Lemma 2. DolevR solves the reliable communication problem with $O(f)$ delivery complexity.

Proof. The target process $p_{t}$ of a content $m$ from $p_{s}$ waits for $f+1$ copies of $m$ coming from distinct paths in $\Pi_{s, t}$. Every time a new copy is received, a $O(1)$ procedure is executed. It follows that the delivery complexity of the protocol is $O(f)$.

The delivery complexity and message complexity of DolevR are optimal solving the reliable communication problem in the system model we consider.

Theorem 1. DolevR solves the reliable communication problem in routed networks assuming the globally bounded Byzantine failure model with optimal message complexity and optimal delivery complexity.

Proof. Given Lemmas 1 and 2, we show that no algorithm can solve the reliable communication problem, in the settings considered in this paper, with an asymptotically lower complexity without considering additional assumptions.

Let us consider two processes $p_{s}$ and $p_{t}$, not connected by a link, respectively as source and target of a reliable communication instance.

The target process relies on the messages it receives from its neighbors to deliver a content. Nevertheless, up to $f$ of its neighbors could be Byzantine faulty and process $p_{t}$ cannot identify them. Thus, a $O(f)$ procedure is required.

Given that $p_{s}$ and $p_{t}$ are not linked, a content must be relayed over faultfree paths (i.e. not including any faulty process) to achieve liveness of reliable communication. In the worst-case scenario the length of the longest fault-free path is $n-k$. A graphical example is provided in Figure 1 .

\subsection{Dolev Topology Unaware Protocol (DolevU)}

DolevU protocol solves the reliable communication problem in unknown networks [7, where contents are flooded in the system. Specifically, DolevU spreads messages $\langle m, p a t h\rangle$, in which $m$ is the content and path is a list data structure collecting the identifier of processes that are traversed by $m$. The source process starts the communication multicasting to all of its neighbors the content $m$ with an empty path. Then, every process $p_{i}$ that receives a message $\langle m$, path $\rangle$ from a neighbor $p_{j}$ adds the identifier of $p_{j}$ to path, it stores $\langle m, p a t h+\{j\}\rangle$ and it relays such a message to all of its neighbors not yet included in path $+\{j\}$. Every process that succeeds identifying $f+1$ disjoint path among the ones it received with a content $m$ delivers $m$.

Protocol complexity. The message complexity of DolevU is factorial in the size of the network, whereas a NP-Complete problem has to be solved verifying every content, as detailed in the following Lemmas. 


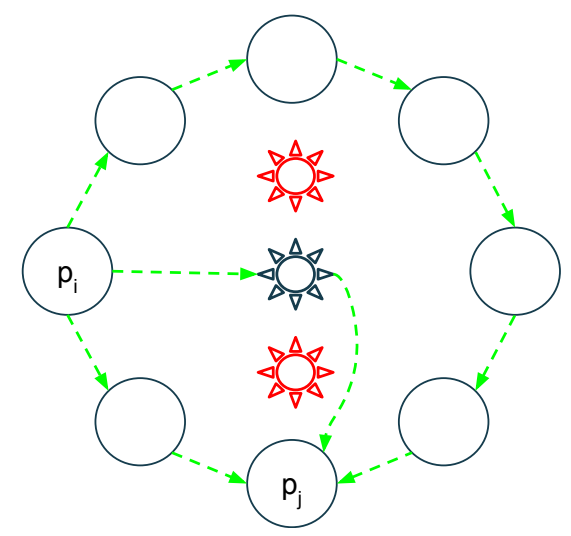

Fig. 1: Explanatory example of Theorem 1 generalized wheel $W(3,8), p_{i}$ and $p_{j}$ are respectively source and target of a reliable communication instance, the sun-shaped nodes are the ones in $K_{3}$. The nodes on top and bottom of $K_{3}$ are faulty.

Lemma 3. DolevU solves the reliable communication problem with a message complexity factorial in the number of processes.

Proof. The content exchanged with DolevU are flooded in the system, collecting the identifier of the traversed processes. Specifically, every received content is relayed by a process to every of it neighbors not yet visited. It follows that every possible path interconnecting a source with any other process is traversed, each generating one message. It follows that the message complexity of DolevU is factorial in the size of the network.

Lemma 4. DolevU solves the reliable communication problem with a NP delivery complexity.

Proof. Currently, the only Byzantine fault-tolerant methodology available to verify whether at least $f+1$ disjoint paths exist among all the ones traversed by a process is the reduction to a NP-Complete problem, set packing [10. It follows that the delivery complexity of DolevU is NP.

The DolevU protocol has been recently reviewed to reduce its message complexity [3. It has been proven that modifications can be adopted in the protocol preventing some messages to be generated. Nonetheless, it is still an open problem whether it is always possible to solve reliable communication in unknown networks, under the weakest assumptions identified by Dolev (Remark 1), with a protocol having polynomial message complexity and/or polynomial delivery complexity. For sake of simplicity, we do not employ the reliable communication protocol defined in [3], given that its worst-case delivery complexity and message complexity is unchanged with respect DolevU.

The DolevU protocol provides the following additional guarantee in case local broadcast links are assumed. 
Theorem 2. Let DolevU solve reliable communication in a network $G$ with local broadcast links. Then, a content $m$ is delivered by every correct process if it is delivered by any correct one.

Proof. When the reliable communication necessary correctness condition is met (Remark 11), the DolevU protocol guarantees that if the source $p_{s}$ of a content $m$ is correct, then any correct target eventually delivers $m$. This is not guaranteed in case of a faulty source: it may diverge from the protocol and it may prevent some targets from delivering its contents. The local broadcast links provide an additional guarantee: every message a process sends is received by all its neighbors. A correct source in DolevU multicast message $\langle m, \emptyset\rangle$ to all of its neighbors. It follows that if a correct process delivered $m$, then message $\langle m, \emptyset\rangle$ has been sent to all neighbors of $p_{s}$, given the local broadcast links, and the claim follows.

\section{Explorer}

Nesterenko and Tixeuil analyzed the Byzantine fault-tolerant topology reconstruction problem [16]. Among the results they provided, two impossibilities have been identified.

Remark 2. No algorithm can decide whether a two-faulty edge exists [16].

Remark 3. No algorithm can compute a reconstruction of only real nodes and edges while including both all correct and all one-faulty edges [16].

They also defined Explorer, an algorithm that enables processes to partially reconstruct the topology of $G$ in the globally bounded failure model assuming KN. It is specified only by the following two procedures: every process $p_{i} 1$ ) broadcasts its neighborhood $\Gamma(i)$ (namely it broadcasts the identifier of processes it has a link with) and 2) it stores all neighborhoods $\Gamma(j)$ delivered with a reliable communication primitive in a dictionary data structure $c T o p_{i}:=\bigcup\langle j, \Gamma(j)\rangle$.

We introduce a simple neighborhood discovery procedure to cope with the unknown neighborhood scenario, defined by the following actions: 1) every process multicasts a HELLO message (basically a message with no payload), and 2) every process that receives a $H E L L O$ message adds the identifier of the sender to its neighborhood.

Then, every process $p_{i}$ broadcasts with a reliable communication primitive its neighborhood $\Gamma(i)$ every time that it changes, and it updates the entry $\langle j, \Gamma(j)\rangle \in c T o p_{i}$ if $\left\langle j, \Gamma(j)^{\prime}\right\rangle$, such that $\Gamma(j) \subset \Gamma(j)^{\prime}$, is delivered.

Additionally, if local broadcast links are assumed, every process $p_{i}$ that delivers two neighborhood $\Gamma(j)$ and $\Gamma(j)^{\prime}$ from $p_{j}$, such that $\Gamma(j)^{\prime} \not \subset\left(\Gamma(j) \in c T o p_{i}\right)$ and $\left(\Gamma(j) \in c T o p_{i}\right) \not \subset \Gamma(j)^{\prime}$, do not consider $j$ for the reconstruction.

Every process $p_{i}$ computes the reconstruction $G_{i}\left(P_{i}, E_{i}\right)$ from $c T o p_{i}$ as follows:

- $\forall\langle u, \Gamma(u)\rangle \in c T o p_{i} \Rightarrow \exists u \in P_{i}$

- $\forall\langle v, \Gamma(v)\rangle,\langle u, \Gamma(u)\rangle \in c T o p_{i}, u \in \Gamma(v) \Rightarrow \exists(v, u) \in E_{i}$. 
- $\forall v \in \Gamma(u),\langle u, \Gamma(u)\rangle \in c$ Top $: X \leftarrow \bigcup u,|X|>f \Rightarrow \exists v \in P_{i}$.

We report some properties of any reconstructed topology $G_{i}$ computed with the defined protocol.

Property 1. (From [16]) $j \notin P \Rightarrow j \notin P_{i}$ (no $G_{i}$ contains non-existent nodes).

Proof. A node $j$ needs to send its neighborhood through a reliable communication primitive or it has to be declared in the neighborhood of at least $f+1$ other nodes to be part of the reconstruction. Given the assumptions of reliable and authenticated links and a reliable communication primitive, none of the two conditions are realizable for a not existing node.

Property 2. Assuming the unknown neighborhood assumption (UN), some reconstruction $G_{i}$ may never include some Byzantine processes.

Proof. In the unknown neighborhood assumption processes are unaware about the peers they have a link with. It follows that a Byzantine neighbor of a process may make itself not discoverable, simply remaining silent. Furthermore, it may adopt such a behavior only with part of its neighbors. It follows that some reconstruction $G_{i}$ may never include some Byzantine processes.

Property 3. (From [16]) Assuming the known neighborhood assumption (KN), the reconstruction $G_{i}$ eventually guarantees the following property: $j \in P_{i} \Leftrightarrow$ $p_{j} \in P$ (Property $1+$ all real nodes are eventually detected).

Proof. All correct processes declare their complete neighborhood assuming KN, therefore every Byzantine process is included in the neighborhood of at least $f+1$ correct nodes due the assumption on the node connectivity of the network.

Property 4. $\forall\langle u, v\rangle \in E, u, v \in$ Correct $\Rightarrow \exists\langle u, v\rangle \in E_{i}$ (all correct edges are eventually contained in $G_{i}$ ).

Proof. All sets of assumptions we consider in our settings enable all processes to reconstruct a topology $G_{i}$ that contains all correct edges. Indeed, the correct edges are declared by both of their endpoints through a reliable communication primitive.

Property 5. $\forall\langle u, v\rangle \in E_{i},\langle u, v\rangle \notin E \Rightarrow u \in$ Byzantine (every spurious edge contains at least one Byzantine process).

Proof. A spurious edge can only be declared by a Byzantine process. Indeed, due to the authenticated channels, correct processes only declare neighbors they have a link with. Given that the neighborhood information is exchanged through a reliable communication primitive, only a Byzantine endpoint of a spurious edge can declare it.

Property 6. (From [16]) Assuming the known neighborhood assumption (KN): $\forall\langle u, v\rangle \in E, u \in$ Correct, $v \in$ Byzantine $\Rightarrow \exists\langle u, v\rangle \in E_{i}$ (all one-faulty edges will eventually be present in any $G_{i}$ ). 
Property \%. Assuming local broadcast links (LBL), all one-faulty edges between a Byzantine process and all of its correct neighbors are eventually either all or none present in every $G_{i}$.

Proof. In case of known neighborhood it is guaranteed by the assumption.

In case of unknown neighborhood, the local broadcast links guarantee that if a neighbor of a process $p_{i}$ receives a message, then it is received by all correct neighbors of $p_{i}$. It follows that if a correct neighbor of $p_{i}$ is detected by $p_{i}$, the same occurs also for all of its correct neighbors.

Property 8. Assuming local broadcast links (LBL), all correct processes eventually share the same topology reconstruction.

Proof. Given Theorem 2, if a correct process delivers a content from $p_{i}$, then all other correct processes deliver it. It follows that all correct processes deliver the same set of messages, even if in potentially different order. The additional rules introduced in Explorer allow processes to eventually share the same topology reconstruction: in an execution of only correct processes, the processes may diffuse many neighborhood declarations, but they are all one superset the other. It follows that all correct processes eventually set the biggest superset they received as neighborhood of every process. Any neighborhood declaration that does not match such an inclusion dependency has necessarily be generated by a Byzantine process, and it is eventually detected by all correct processes (Theorem 2).

Property 9. No reconstruction $G_{i}$ computed assuming local broadcast links (LBL) will ever contain more real edges than one obtained assuming the known neighborhood assumption (KN).

Proof. It follows from Properties 3 and 7 . Furthermore, any reconstruction built assuming KN eventually includes all correct and one-faulty edges, whereas some one-faulty edges may miss assuming UN and LBL.

\subsection{Protocol Complexity Analysis}

All correct processes $p_{i}$ in Explorer broadcast their neighborhood $\Gamma(i)$. Supposing the know neighborhood assumption $(\mathrm{KN})$, every process broadcasts such information only once. It follows that Explorer requires $\mathcal{O}(n)$ reliable communication executions to enable all correct processes to compute $G_{i}$. Considering the unknown neighborhood (UN) assumption, every process has to perform the neighborhood discovery and then to broadcast its $\Gamma(i)$. Unfortunately, no process $p_{i}$ knows how many nodes have to be detected before diffusing $\Gamma(i)$, and thus, they may broadcast their neighborhood many times, $n-f-1$ in the worst-case scenario. It follows that Explorer with neighborhood discovery executes $\mathcal{O}\left(n^{2}\right)$ reliable communication instances to enable all correct processes to compute $G_{i}$. 


\subsection{Fault-free Disjoint Path Solution}

The Explorer protocol enables processes to partially reconstruct the topology of $G$. We showed that different sets of assumptions provide more or less accurate reconstructions (Properties 1 19 ). We reported the DolevR protocol, that it leverages disjoint routes defined between all pairs of processes to achieve reliable communication. We highlighted how $f$ Byzantine faulty processes may compromise at most $f$ paths of any disjoint path solution $\Pi_{i, j}$ in DolevR, and that the liveness of such a protocol is guaranteed by the existence of disjoint path solutions of size greater than $2 f$ between all pairs of processes, where at least $f+1$ paths cannot be compromised. It follows that, if every pair of correct processes is able to identify a disjoint path solution interconnecting them where at least $f+1$ paths are faults-free (i.e. they do not include any Byzantine faulty process), real and disjoint $\left(F F_{-} R_{-} D\right)$, then they are able to achieve reliable communication.

We analyze several sets of assumptions that enable all pairs of correct processes $p_{i}, p_{j}$ to compute a disjoint path solutions $\Pi_{i, j}$ in $G_{i}$ containing at least $f+1$ FF_R_D paths.

Theorem 3. The set of assumptions a) $\mathbf{k}>\mathbf{3} \mathbf{f}$, b) unicast links and c) unknown neighborhood enables every correct process $p_{i}$ to compute a disjoint paths solution $\Pi_{i, j}$ toward any correct process $p_{j}$ that contains at least $f+1$ faults-free, real and disjoint paths.

Proof. Let us assume processes employing Explorer and that all messages it generates have been already delivered by the peers. The unknown neighborhood assumption and unicast links allow Byzantine faulty processes to decide which one-faulty and two-faulty edges to declare (Remarks 23), thus the local connectivity between any two processes $p_{i}, p_{j}$ in the reconstructed topology may be reduced by at most $f$. It follows that any disjoint paths solution $\Pi_{i, j}$ will contain more than $2 f$ paths (Property 4). Given that at most $f$ paths of any $\Pi_{i, j}$ may contain faults the claim follows.

Theorem 4. The set of assumptions a) $\mathbf{k} \leq \mathbf{3 f}$, b) unicast link and c) unknown neighborhood is not sufficient to enable every correct process $p_{i}$ to compute a disjoint paths solution $\Pi_{i, j}$ toward every correct process $p_{j}$ containing at least $f+1$ faults-free, real and disjoint paths with any protocol.

Proof. The unknown neighborhood assumption and the unicast links allow faulty processes to decide which one-faulty and two-faulty edges are detectable by correct processes (Remarks 23). It follows that the faulty processes may potentially be able to reduce the local connectivity between some pairs of correct processes $p_{i}, p_{j}$ by $f$ : the local connectivity $\kappa_{i, j}$ in $G_{i}$ may be lower than $2 f$ and at most $2 f-1$ disjoint path $\Pi_{i, j}$ will be identifiable between $p_{i}$ and $p_{j}$, whatever algorithm is envisioned for the reconstruction. Then, up to $f$ paths in $\Pi_{i, j}$ may include faulty processes and the claim follows. A graphical example is provided in Figure2, 

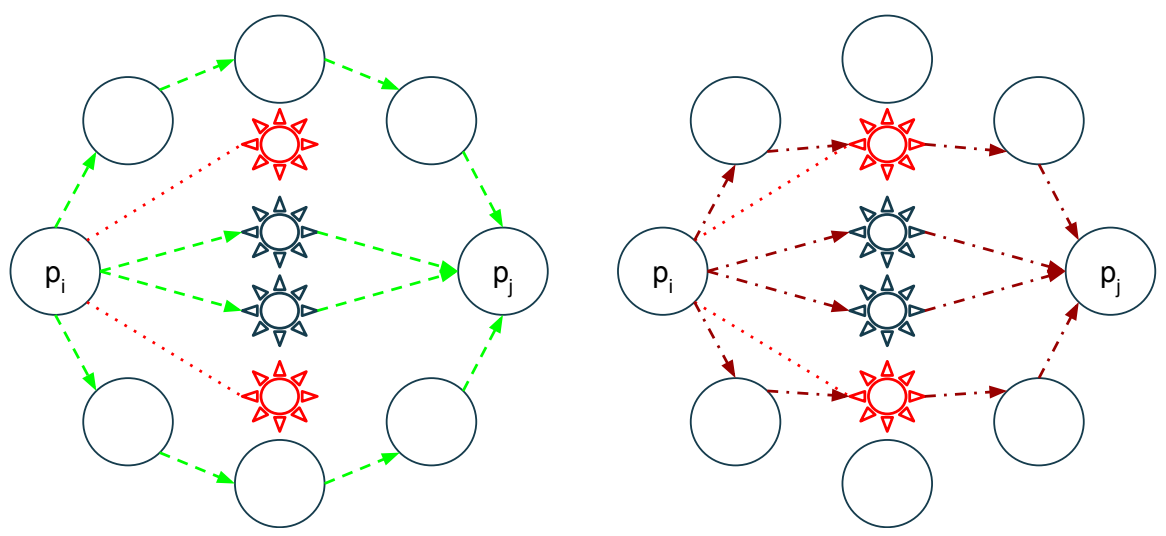

Fig. 2: Explanatory example of Theorem 4 a $W(4,8)$ generalized wheel (6connected graph), the "sun-shaped" nodes are the ones in $K_{4}$. Let us consider $W(4,8)$ as communication network and let us assume that two nodes in $K_{4}$ are Byzantine faulty (thus, $f=2$ and $k=6 \leq 3 f=6$ ); let us select two not adjacent processes $p_{i}$ and $p_{j}$ in the cycle $C_{8}$ respectively as source and target of a reliable communication instance and let us assume that the Byzantine processes hide the edges interconnecting them with $p_{i}$ (dashed edges). After reconstructing the topology, processes compute a solution $\Pi_{i, j}$, but it could contain at least $f+1$ FF_R_D paths (figure on the left) or not (figure on the right).

Theorem 5. The set of assumptions a) $\mathbf{k}>\mathbf{2} \mathbf{f}+\lfloor\mathbf{f} / \mathbf{2}\rfloor$, b) local broadcast links and c) unknown neighborhood enables every correct process $p_{i}$ to compute a disjoint paths solution $\Pi_{i, j}$ toward any correct process $p_{j}$ containing at least $f+1$ faults-free, real and disjoint paths.

Proof. Given Property 7 , let us suppose that $f_{d} \leq f$ Byzantine processes decide to be detected by their neighbors and they send the HELLO message, whereas $f-f_{d}$ ones do not. Let us assume that all messages exchanged by Explorer have been already delivered and let us consider $\Pi_{i, j}$ as the disjoint path solution computed on $G_{i}$ between a pair of correct processes $p_{i}$ and $p_{j}$. The assumption on the node connectivity of $G$ guarantees that at least $2 f+\lfloor f / 2\rfloor+1$ disjoint paths exist between $p_{i}$ and $p_{j}$ in the communication network. The undeclared Byzantine processes may reduce the local connectivity between $p_{i}$ and $p_{j}$ by $f-f_{d}$ in $G_{i}$. Let us temporarily assume, for the purpose of the proof, that the declared Byzantine processes behave as correct ones. It follows, from Property 4 and 7 of Explorer, that the size of $\Pi_{i, j}$ would be at least equal to:

$$
2 f+\lfloor f / 2\rfloor+1-\left(\mathbf{f}-\mathbf{f}_{\mathbf{d}}\right)=f+\lfloor f / 2\rfloor+1+f_{d}
$$

Specifically, all paths between $p_{i}$ and $p_{j}$ that contain only correct or declared Byzantine processes existing in $G$ are present in $G_{i}$.

Let us now consider the declared Byzantine processes not reporting the edges existing between them (i.e. the two-faulty edges). It follows that the paths in $G$ 
containing two-faulty edges may not be present in $G_{i}$ (Remark 2). Therefore, pairs of Byzantine processes may potentially cause a reduction to the maximum size of $\Pi_{i, j}$ : every couple may decrease the number of available disjoint paths in $G_{i}$ between $p_{i}$ and $p_{j}$ by one. It follows that the size of $\Pi_{i, j}$ would be at most reduced to:

$$
f+\lfloor f / 2\rfloor+1+f_{d}-\left\lfloor\mathbf{f}_{\mathbf{d}} / \mathbf{2}\right\rfloor
$$

namely, $f_{d}$ declared Byzantine faulty processes may reduce the local connectivity between $p$ and $q$ in $G_{i}$ by at most $\left\lfloor f_{d} / 2\right\rfloor$. The $f_{d}$ declared Byzantine processes may also be selected in the paths $\Pi_{i, j}$. Specifically, in the worst case scenario $f_{d}$ paths in $\Pi_{i, j}$ may contain Byzantine processes. It follows that at most $f_{d}$ paths would not be fault-free, and thus the remaining fault-free ones in $\Pi_{i, j}$ would be:

$$
f+\lfloor f / 2\rfloor+1+f_{d}-\left\lfloor f_{d} / 2\right\rfloor-\mathbf{f}_{\mathbf{d}}=f+1+\lfloor f / 2\rfloor-\left\lfloor f_{d} / 2\right\rfloor
$$

Thus, at least $f+1$ paths in $\Pi_{i, j}$ are faults-free, real and disjoint.

Notice that, given Property 9 , the Theorem 5 extends substituting local broadcast links with the unicast ones and assuming the known neighborhood assumption.

\section{CombinedRC, Reliable Communication Protocol}

We combine Explorer, DolevU and DolevR protocols to design a new reliable communication primitive. We call such a protocol CombinedRC, that aims to set up an efficient reliable communication service.

The Explorer protocol is used to partially reconstruct the network topology, and then to enable processes to compute disjoint paths solutions through which relay contents. The DolevU protocol is adopted as reliable communication subprimitive by Explorer and CombinedRC during the initialization. Lastly, the DolevR protocol is employed as actual reliable communication primitive in CombinedRC, leveraging the routes computed and communicated using Explorer and DolevU.

We showed in Section 5.2 that Explorer, under certain conditions, enables every correct process $p_{i}$ to identify a disjoint paths solution $\Pi_{i, j}$ interconnecting it with any other correct process $p_{j}$, such that at least $f+1$ paths in the solution are faults-free, real and disjoint. Once that the solution $\Pi_{i, j}$ is known to both $p_{i}$ and $p_{j}$, they can efficiently communicate. We claimed in Property 8 that all correct processes eventually obtain the same topology reconstruction in case local broadcast links are employed. Thus, under such an assumption, processes $p_{i}$ and $p_{j}$ eventually compute the same solution $\Pi_{i, j}$. Under the weaker condition of unicast links, the reconstructed topologies may differ on distinct processes, thus a source process $p_{i}$ has additionally to communicate the computed solution $\Pi_{i, j}$ to a target process $p_{j}$ using DolevU.

Any source process $p_{i}$ routes contents through the computed $\Pi_{i, j}$ and any target process $p_{j}$ waits for messages over $f+1$ paths among the ones in $\Pi_{i, j}$. 
The pseudo-code of CombinedRC is presented in Algorithm 1 .

Every process relays its contents over the computed routes if available, otherwise, they are queued for subsequent transmission (lines 1 1 5).

Every process $p_{i}$ attempts to compute a solution $\Pi_{i, j}$ toward every other process $p_{j}$ of the system. In the case of local broadcast links, the reconstructed topology $G_{i}$ is eventually the same in every process. Therefore, a source process has to relay its contents over the computed disjoint routes every time they change (a finite number of times). In case of unicast links, once that the local connectivity toward a target $p_{j}$ reaches a value greater than $2 f$, the source process $p_{i}$ communicates the computed solution $\Pi_{i, j}$ via DolevU (lines 6.21).

Every process relays contents or computed disjoint solution following the path attached to messages (lines 22 33).

Every process that delivers a disjoint paths solution with DolevU adopts it to verify contents (lines $34 \quad 35)$ using DolevR (lines 36 37).

\subsection{CombinedRC Correctness}

Theorem 6. CombinedRC provides safety of reliable communication.

Proof. A process that receives a $C N T$ message checks the identity of the sender through authenticated links. It follows that any spurious CNT message will contain in the path field at least one identifier of the Byzantine processes. It follows that the faulty processes cannot diffuse $C N T$ messages over more than $f$ disjoint paths.

Theorem 7. CombinedRC provides liveness of reliable communication in all cases where Explorer succeeds in identifying a disjoint path solution between two processes $i, j$ that contains $f+1 F F_{-} R_{-} D$ paths.

Proof. Let us assume that all messages exchanged by Explorer have been already delivered and that a process $p_{i}$ aims to reliably communicate with a correct process $p_{j}$. In case of local broadcast links, processes $p_{i}$ and $p_{j}$ eventually share the same topology reconstruction $G_{i}$, thus also the disjoint path solution $\Pi_{i, j}$ will eventually be the same both on $p_{i}$ and $p_{j}$. Process $p_{i}$ relays the contents through $\Pi_{i, j}$ every time such a solution changes. The assumption of $f+1$ FF_R_D paths in $\Pi_{i, j}$ guarantees reliable communication. In case of unicast channels, the solution $\Pi_{i, j}$ is diffused via DolevU and contents are routed over $\Pi_{i, j}$. The assumption of $f+1$ FF_R_D paths in $\Pi_{i, j}$ guarantees reliable communication.

\subsection{Protocol Complexity Analysis}

CombinedRC provides reliable communication with optimal message complexity and delivery complexity (Theorem 11). Specifically, it routes contents over computed disjoint routes as DolevR, thus $\mathcal{O}(n)$ messages per content are exchanged, and an $\mathcal{O}(f)$ procedure is executed to verify any content.

CombinedRC requires an initialization phase where the network topology is partially reconstructed and the solutions containing $f+1$ FF_R_D paths are 


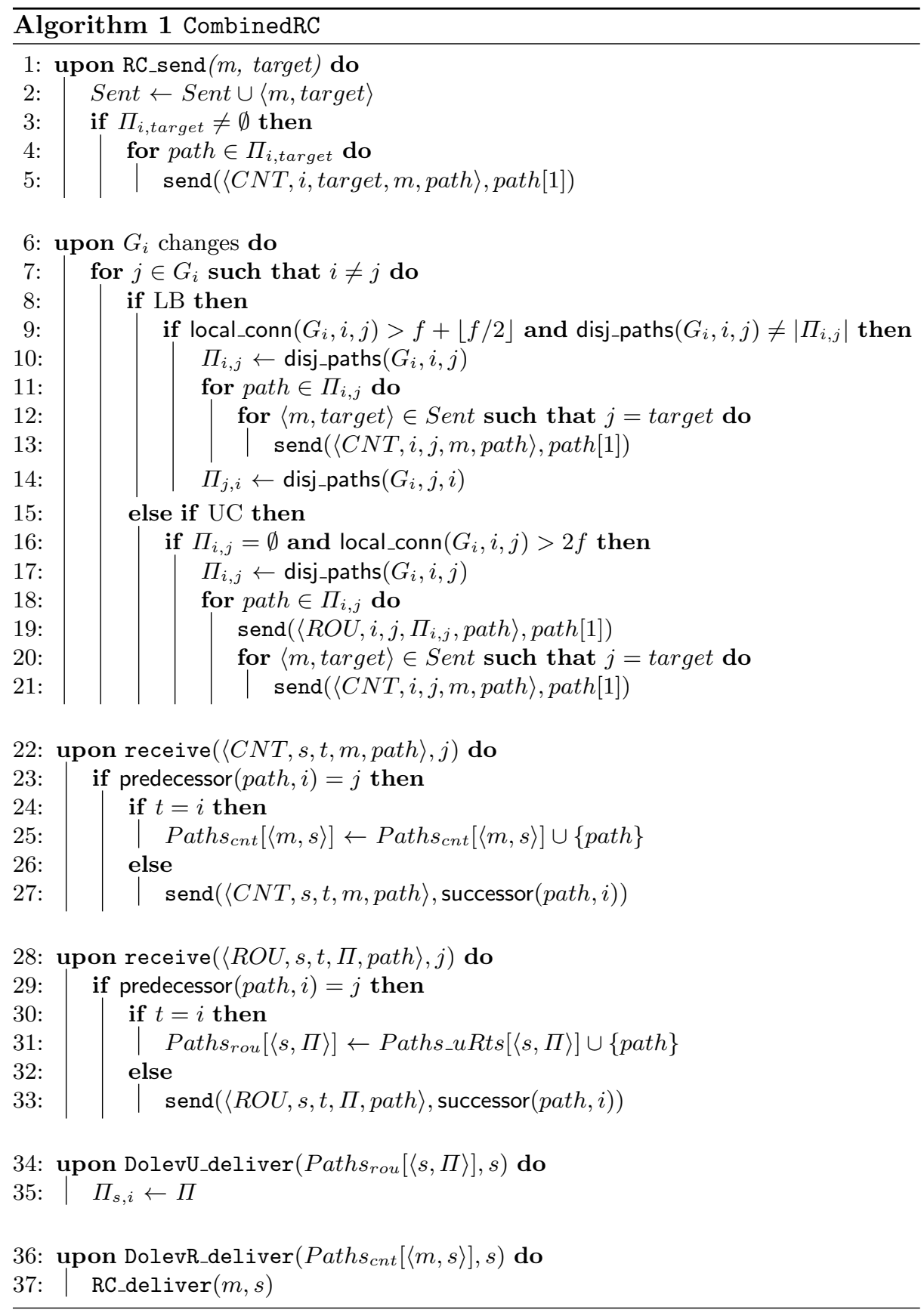


computed between every pair of correct processes. We showed in Section 5 that Explorer requires at most $\mathcal{O}\left(n^{2}\right)$ reliable communication instances to partially reconstruct the network topology. The same solution $\Pi_{i, j}$ is eventually computed by both $p_{i}$ and $p_{j}$, assuming local broadcast channels, without additional message exchanges, because the topology reconstruction will eventually be the same on every process and the disjoint paths solutions can be computed through a deterministic algorithm. On the other hand, employing unicast links, every couple of processes has to agree on a solution $\Pi_{i, j}$. Thus, an additional content exchange (with payload $\Pi_{i, j}$ ) using a reliable communication primitive has to be performed for each pair of correct processes. It follows that the initialization phase of CombinedRC requires the execution of $\mathcal{O}\left(n^{2}\right)$ DolevU instances. Notice that, in the case of known neighborhood and local broadcast links, the cost of the initialization phase reduces to $\mathcal{O}(n)$ DolevU instances, indeed each process diffuses its neighborhood only once and all correct processes eventually share the same reconstruction.

\section{Conclusion}

We demonstrated how to boost the efficiency of reliable communication despite some of the participants being Byzantine faulty, when the network topology is unknown to the participants, assuming reliable authenticated links. Our solution combines a costly topology reconstruction process, that is executed once, and an efficient reliable communication scheme that is optimal both in terms of exchanged messages and of local computation complexity. Without leveraging the topology reconstruction, the cost of every reliable communication instance in the same scenario would have been factorial in message complexity and NP in delivery complexity.

An interesting path for future research is to decrease the adversary capabilities. A noteworthy candidate is the computationally bounded adversary, that enables solutions based on cryptography.

\section{References}

1. Bhandari, V., Vaidya, N.H.: Implementing a reliable local broadcast primitive in wireless ad hoc networks. https://disc.georgetown.domains/publications/ rbcast-tech.pdf

2. Bhandari, V., Vaidya, N.H.: On reliable broadcast in a radio network. In: Aguilera, M.K., Aspnes, J. (eds.) Proceedings of the Twenty-Fourth Annual ACM Symposium on Principles of Distributed Computing, PODC 2005, Las Vegas, NV, USA, July 17-20, 2005. pp. 138-147. ACM (2005). https://doi.org/10.1145/1073814.1073841, https://doi.org/10.1145/1073814. 1073841

3. Bonomi, S., Farina, G., Tixeuil, S.: Multi-hop byzantine reliable broadcast with honest dealer made practical. J. Braz. Comp. Soc. 25(1), 9:1-9:23 (2019). https://doi.org/10.1186/s13173-019-0090-x 
4. Cachin, C., Guerraoui, R., Rodrigues, L.E.T.: Introduction to Reliable and Secure Distributed Programming (2. ed.). Springer (2011). https://doi.org/10.1007/9783-642-15260-3

5. Diestel, R.: Graph Theory. Springer Berlin Heidelberg (2017). https://doi.org/10.1007/978-3-662-53622-3

6. Dinic, E.A.: Algorithm for solution of a problem of maximum flow in networks with power estimation. In: Soviet Math. Doklady. vol. 11, pp. 1277-1280 (1970)

7. Dolev, D.: Unanimity in an unknown and unreliable environment. In: 22nd Annual Symposium on Foundations of Computer Science, Nashville, Tennessee, USA, 2830 October 1981. pp. 159-168 (1981). https://doi.org/10.1109/SFCS.1981.53

8. Dolev, S., Liba, O., Schiller, E.M.: Self-stabilizing byzantine resilient topology discovery and message delivery - (extended abstract). In: Networked Systems - First International Conference, NETYS 2013, Marrakech, Morocco, May 2-4, 2013, Revised Selected Papers. pp. 42-57 (2013). https://doi.org/10.1007/978-3-642-40148$0 \_4$

9. Edmonds, J., Karp, R.M.: Theoretical improvements in algorithmic efficiency for network flow problems. J. ACM 19(2), 248-264 (1972). https://doi.org/10.1145/321694.321699

10. Garey, M.R., Johnson, D.S.: Computers and Intractability; A Guide to the Theory of NP-Completeness. W. H. Freeman \& Co., New York, NY, USA (1990)

11. Khan, M.S., Naqvi, S.S., Vaidya, N.H.: Exact byzantine consensus on undirected graphs under local broadcast model. In: Robinson, P., Ellen, F. (eds.) Proceedings of the 2019 ACM Symposium on Principles of Distributed Computing, PODC 2019, Toronto, ON, Canada, July 29 - August 2, 2019. pp. 327336. ACM (2019). https://doi.org/10.1145/3293611.3331619, https://doi.org/ $10.1145 / 3293611.3331619$

12. Maurer, A., Tixeuil, S.: Byzantine broadcast with fixed disjoint paths. J. Parallel Distrib. Comput. 74(11), 3153-3160 (2014). https://doi.org/10.1016/j.jpdc.2014.07.010

13. Maurer, A., Tixeuil, S.: Containing byzantine failures with control zones. IEEE Trans. Parallel Distrib. Syst. 26(2), 362-370 (2015). https://doi.org/10.1109/TPDS.2014.2308190

14. Maurer, A., Tixeuil, S.: Tolerating random byzantine failures in an unbounded network. Parallel Processing Letters 26(1) (2016). https://doi.org/10.1142/S0129626416500031

15. Menger, K.: Zur allgemeinen kurventheorie. Fundamenta Mathematicae 10(1), 96115 (1927)

16. Nesterenko, M., Tixeuil, S.: Discovering network topology in the presence of byzantine faults. IEEE Trans. Parallel Distrib. Syst. 20(12), 1777-1789 (2009). https://doi.org/10.1109/TPDS.2009.25

17. Pagourtzis, A., Panagiotakos, G., Sakavalas, D.: Reliable broadcast with respect to topology knowledge. Distributed Computing 30(2), 87-102 (2017). https://doi.org/10.1007/s00446-016-0279-6

18. Pelc, A., Peleg, D.: Broadcasting with locally bounded byzantine faults. Inf. Process. Lett. 93(3), 109-115 (2005). https://doi.org/10.1016/j.ipl.2004.10.007

19. Xu, J.: Topological structure and analysis of interconnection networks, vol. 7 . Springer Science \& Business Media (2013)

20. Zeng, K., Govindan, K., Mohapatra, P.: Non-cryptographic authentication and identification in wireless networks. IEEE Wireless Commun. 17(5), 56-62 (2010). https://doi.org/10.1109/MWC.2010.5601959 